\title{
Dietas vegetarianas en alumnos universitarios
}

\author{
Vegetarian diets in first year university students
}

\author{
Javiera Aravena ${ }^{\mathrm{a}}$, Tamara Zubarew ${ }^{\mathrm{b}}$, Paula Bedregal ${ }^{\mathrm{c}}$, Soledad Zuzulich ${ }^{\mathrm{d}}$, Pascuala Urrejola ${ }^{\mathrm{b}}$
}

aBecado de Pediatría, Pontificia Universidad Católica de Chile. Santiago, Chile

bDivisión de Pediatría, Unidad de Adolescencia, Pontificia Universidad Católica de Chile. Santiago, Chile

‘Departamento de Salud Pública, División de Salud Pública y Medicina Familiar, Pontificia Universidad Católica de Chile. Santiago, Chile

dEnfermera Matrona, Dirección de Asuntos Estudiantiles, Pontificia Universidad Católica de Chile.

Recibido: 25 de marzo de 2020; Aceptado: 25 de junio de 2020

¿Qué se sabe del tema que trata este estudio?

Las dietas vegetarianas han aumentado en popularidad y cada vez en edades más precoces. Se presentan como saludables, pero si no son balanceadas se pueden asociar a déficits de calcio, ácidos grasos omega 3 y vitamina B12 entre otros.

\section{¿Qué aporta este estudio a lo ya conocido?}

Existe un elevado porcentaje de vegetarianos entre los universitarios encuestados. Un tercio comenzó entre los 12 y 18 años. El 75,7\% obtiene información sobre su dieta a través de medios digitales. Solo la mitad estaba suplementado con vitamina B 12 .

\section{Resumen}

Se ha observado un aumento en la popularidad de las dietas vegetarianas, en especial en la población adolescente y adulto joven. Estas dietas se presentan como saludables y balanceadas pero las recomendaciones son controversiales en cuanto a los potenciales déficit nutricionales. Objetivo: Identificar la frecuencia y tipos de dieta vegetariana utilizada, su motivación y fuentes de información. Pacientes y Método: Estudio transversal y analítico en universitarios de primer año de la Pontificia Universidad Católica de Chile (PUC). Se realizó una encuesta vía online obteniendo información demográfica y caracterización de dietas de aquellos que se consideraban vegetarianos. Las variables fueron analizadas mediante el software IDM SPSS Statistics ${ }^{\circledR}$ y en planilla Excel ${ }^{\circledR}$ de forma cuantitativa. Resultados: 152 alumnos respondieron la encuesta (15,2\% del total), siendo el $49,4 \%$ de estos vegetariano. El 32,4\% inició este patrón alimentario entre los 12-18 años; sus motivaciones más frecuentes fueron las medio ambientalistas $(91,9 \%)$ y animalistas (72,9\%). El 52,9\% de los vegetarianos reciben suplementación de vitamina b 12 pero solo un 15,9\% reportó presentar este déficit. El 75,7\% obtiene información relacionada acerca de las dietas vegetarianas a través de medios digitales. Conclusión: Se encontró un alto porcentaje de estudiantes vegetarianos en las encuestas contestadas, lo que hace necesario que los profesionales de salud estén capacitados en esta área para asegurar una adecuada educación nutricional, suplementación si es necesario, y seguimiento.

Correspondencia:

Pascuala Urrejola

purrejol@med.puc.cl 


\begin{abstract}
There has been an increase in the popularity of vegetarian diets, especially among adolescents and young adults. These diets seem to be healthy and balanced, but the recommendations are controversial regarding potential nutritional deficits. Objective: To identify the frequency and types of vegetarian diet used, their motivation, and sources of information. Patients and Method: Cross-sectional analytical study in freshmen students from the Pontifical Catholic University of Chile (PUC). Through an online survey, we collected demographic information and diet characterization of those who considered themselves as vegetarians. Variables were analyzed using IDM SPSS Statistics ${ }^{\circledR}$ software and in Excel ${ }^{\circledR}$ spreadsheet in a quantitative way. Results: 152 students answered the survey ( $15.2 \%$ of the sample) and, out of these, $49.4 \%$ were vegetarian. $32.4 \%$ started this eating pattern between the ages 12 and 18 and among their most frequent motivations were environmentalists (91.9\%) and animalists (72.9\%). 52.9\% of vegetarians take vitamin B 12 supplementation but only $15.9 \%$ reported having this deficiency. $75.7 \%$ obtain information related to vegetarian diets through digital media. Conclusion: In the surveys answered, we found a high percentage of vegetarian students, therefore, health professionals need to be trained in this area to assure adequate nutritional education, supplementation if necessary, and follow-up.
\end{abstract}

Keywords:

Diet;

Vegetarian;

Vegan;

Adolescents

\section{Introducción}

El vegetarianismo es un término amplio que abarca una variedad de prácticas alimentarias. Se define como vegetariano a aquella persona que excluye los alimentos de origen animal o parte de ellos, los veganos en cambio no consumen ningún producto de origen animal ni sus derivados (Tabla 1) ${ }^{1-3}$. En los últimos años ha aparecido el concepto de "flexitarianismo", que es la práctica de ser «flexible» sobre la forma en que se es vegetariano. Un flexitariano práctica una dieta vegetariana pero, consume productos de origen animal una o dos veces por semana $a^{4,5}$.

Existen múltiples razones para adoptar una dieta vegetariana incluyendo razones éticas, espirituales o religiosas como también, la preocupación por el bienestar animal o por razones medio ambientales ${ }^{5-9}$. Esto último debido a la divulgación cada vez más frecuente, en medios de comunicación social, de estudios que evaluan el impacto sobre el medio ambiente y sobre los recursos hídricos de la producción intensiva de alimentos de origen animal ${ }^{10}$. Una dieta vegetariana también puede ser parte de un estilo de vida o de identidad ${ }^{1,2}$. La decisión de hacer una dieta vegetariana por razones de salud, puede estar influida por la percepción de los beneficios en la salud que aparecen frecuentemente en los medios de comunicación, como también por el número creciente de estudios epidemiológicos que muestran una asociación entre este tipo de dietas y la prevención y tratamiento de algunas enfermedades crónicas no transmisibles y de algunos tipos de cáncer ${ }^{9,11-13}$.

A pesar de lo anterior, este patrón alimentario puede resultar en una baja biodisponibilidad de determinados micronutrientes tales como hierro, zinc y selenio, o déficit de vitamina B12, vitamina D y de ácidos grasos poliinsaturados omega 3 (n-3). Esto se asocia a riesgo de déficit nutricional, por lo que se hace necesaria una adecuada supervisión y educación nutricional ${ }^{5,8,10,12}$.

En los últimos años se ha observado un aumento en la popularidad de las dietas vegetarianas, en especial en la población adolescente y adulto joven ${ }^{1,2,9,14}$. Estudios recientes han mostrado que un 3,3 a 5\% de los adultos y niños en Estados Unidos se reportan como vegetarianos y el 3,7\% como veganos ${ }^{3,4}$. En Europa la prevalencia de vegetarianos llega a un $5 \%{ }^{15}$. En Chile, no existen datos epidemiológicos acerca de la prevalencia $o$ frecuencia de dieta vegetariana ${ }^{1,14}$.

Diversos estudios han demostrado que a pesar de las posibles implicancias en la salud de una dieta vegetariana, menos del $10 \%$ de los vegetarianos consultan a un profesional de la salud para consejería nutricional. La gran mayoría, en especial los adolescentes y jóvenes, basan su conocimiento en fuentes de información tales como las redes sociales o similares y pocos en artículos con información de buena calidad y profesional ${ }^{1,13}$.

Por todo lo anterior, el objetivo de esta investigación es identificar la frecuencia y tipos de vegetarianos, en estudiantes universitarios de primer año en Santiago Chile, además de su motivación y fuentes de información utilizadas para seguir este tipo de alimentación. Los resultados de este estudio servirán para futuras investigaciones e implementar medidas preventivas para evitar complicaciones de salud a corto y largo plazo.

\section{Pacientes y Método}

Se realizó un estudio transversal descriptivo en universitarios (mayores de 18 años) de primer año de la Pontificia Universidad Católica de Chile (PUC). El 
universo total corresponde a 4.900 alumnos de primer año. Se estimó una muestra basado en la información disponible en la Encuesta Nacional de Medio Ambiente del año $2018^{16}$. De acuerdo con la Encuesta, la proporción de la población que no consume leche o huevos es aproximadamente un 3\%. Considerando un nivel de confianza del 95\%, con una precisión del $2 \%$, se estimó un tamaño de al menos 275 estudiantes. Dado que el método de recolección de la información fue a través de una encuesta enviada por correo electrónico, y que la tasa de respuestas promedio, con este método es entre 10-30\% (dato obtenido de Dirección de Análisis Institucional y Planificación de la PUC), se decide invitar a participar a 1.000 alumnos. La muestra se obtuvo de manera aleatoria a partir de la información de los correos electrónicos de los alumnos de primer año de la PUC.

La encuesta enviada a los estudiantes incluyó información demográfica (sexo, edad, carrera universitaria cursando), consumo alimentario y un área exclusiva para los que se consideraban vegetarianos, donde se preguntó sobre edad de inicio de la dieta, motivaciones por las cuales se optó por esta dieta, antecedentes de visita a profesionales de la salud, padecimiento de alguna condición de salud (déficit nutricional o enfermedad) desde que se inició la dieta, suplementos nutricionales que está consumiendo y las fuentes de información utilizadas.

Se realizó un análisis descriptivo con los datos, estableciendo frecuencias, promedios, rangos y desviación estándar para variables continuas. Se realizaron análisis univariados para identificar diferencias entre grupos. Para esto se utilizó pruebas no paramétricas para identificar diferencias en la homogeneidad de la distribución (Chi2) y diferencias en los promedios (Kruskal Wallis).

Todos los análisis fueron realizados mediante el software IDM SPSS Statistics ${ }^{\circledR}$ (Chicago, IL, USA), versión 20.0 y en planilla Excel ${ }^{\circledR}$.

El estudio fue aprobado por el Comité de Ética de Investigación de la Facultad de Medicina de la Pontificia Universidad Católica de Chile (180318002).

\section{Resultados}

La encuesta fue contestada por 152 alumnos, $86 \%$ de edades entre 18 y 20 años, y 72\% mujeres. No hubo diferencias significativas entre las carreras respecto a predominio de algún patrón alimentario.

El 49,4\% de los encuestados se consideró vegetariano: $21,1 \%$ lacto-ovo-vegetariano, $14,5 \%$ pesco-vegetariano, 4,6\% vegano, 3,9\% flexitariano, 3,3\% lactovegetariano y $2 \%$ ovo-vegetariano. El 7,2\% estaba considerando optar por esta dieta.
De los vegetarianos, el $84 \%$ fueron mujeres entre 18 y 20 años. El 43,2\% inició esta dieta después de los 18 años, un $32,4 \%$ entre los $12-18$ años y solo un 2,7\% antes de los 12 años.

Las motivaciones más frecuentes para optar por una dieta vegetariana fueron las medio- ambientalistas y animalistas (Tabla 2).

Un 33,8\% presentó un déficit nutricional o alguna enfermedad desde el inicio de la dieta (Tabla 3). Un $66 \%$ recibió algún tipo de suplementación (Tabla 4).

Tabla 1. Categorías de Dietas Vegetarianas

\begin{tabular}{ll}
\hline Dieta & Producto de origen animal consumido \\
\hline Lacto-ovo-vegetariano & Sólo consume lácteos y huevo \\
Lacto-vegetariano & Sólo consume lácteos \\
Ovo-vegetariano & Sólo consume huevos \\
Vegano & No consume productos de origen animal ni \\
& sus derivados \\
Pesco-vegetariano & Sólo consume productos de mar \\
Flexitariano & Consume productos de origen animal pero \\
& solo una a dos veces por semanas \\
\hline
\end{tabular}

Tabla 2. Motivaciones en estudiantes vegetarianos. $\mathbf{N}$ total $=\mathbf{7 4}$

\begin{tabular}{lcc}
\hline Motivo & $\mathrm{n}$ & $\%$ \\
\hline Medioambientales & 67 & 91,9 \\
Animales/maltrato animal & 53 & 72,9 \\
Beneficios para la salud & 35 & 47,9 \\
Desagrado por el sabor de la carne & 18 & 24,3 \\
Control de peso & 6 & 8,1 \\
Razones económicas & 2 & 2,7 \\
Creencias religiosas & 1 & 1,4 \\
Inculcado por familia & 0 & 0 \\
\hline
\end{tabular}

$\mathrm{n}$ = número de estudiantes en cada categoría de motivación. \% = porcentaje de estudiantes del total en cada categoría de motivación

Tabla 3. Condiciones de salud en estudiantes desde inicio de la dieta vegetariana. $\mathbf{N}$ total $=\mathbf{7 4}$

\begin{tabular}{lcc}
\hline Condición de salud & $\mathrm{n}$ & $\%$ \\
\hline Ninguna & 48 & 66,2 \\
Déficit vitamina b 12 & 13 & 15,9 \\
Déficit vitamina D & 12 & 16,9 \\
Déficit de hierro & 8 & 11,2 \\
Trastorno de conducta alimentaria & 2 & 2,8 \\
Enfermedad celíaca o Enfermedad Inflamatoria & 1 & 1,4 \\
Intestinal & &
\end{tabular}

$\mathrm{n}=$ Número de estudiantes que declara cada condición de salud. $\%=$ porcentaje de estudiantes del total que declaran cada condición de salud. 
Tabla 4. Suplementos nutricionales utilizados por vegetarianos. $\mathrm{N}$ total $=\mathbf{7 4}$

\begin{tabular}{lcc}
\hline Suplemento nutricional & $\mathrm{n}$ & $\%$ \\
\hline Ninguno & 25 & 34 \\
Vitamina B 12 & 39 & 52,9 \\
Multivitamínico & 17 & 22,9 \\
Vitamina D & 15 & 20 \\
Fierro & 9 & 12,9 \\
Omega 3 & 4 & 5,7 \\
Zinc & 2 & 2,9 \\
Otro & 2 & 2,9 \\
\hline
\end{tabular}

$\mathrm{n}=$ Número de estudiantes que declara consumir cada categoría de suplemento nutricional. $\%=$ porcentaje del total de estudiantes que declara consumir cada categoría de suplemento nutricional.

Tabla 5. Principales fuentes de información utilizadas por vegetarianos. $\mathbf{N}$ total $=\mathbf{7 4}$

\begin{tabular}{lcc}
\hline Fuente de información & $\mathrm{n}$ & $\%$ \\
\hline $\begin{array}{l}\text { Medios Digitales (foros, blogs, páginas web, Facebook, } \\
\text { Instagram) }\end{array}$ & 55 & 75,7 \\
Familia y/o amigos & 34 & 47,3 \\
Profesionales de salud & 33 & 45,9 \\
Libros o Revistas científicas & 28 & 37,8 \\
Medios de Comunicación (televisión, radio, periódicos) & 20 & 27 \\
Ninguno & 6 & 8,1 \\
\hline
\end{tabular}

$\mathrm{n}$ = Número de vegetarianos que señala fuente de información $\%=$ porcentaje del total de vegetarianos que señala dicha fuente de información.

Un $42 \%$ tuvo control con un profesional de la salud (médico o nutricionista): 30\% acudió en una ocasión, $30 \%$ una vez al mes y un $40 \%$ acude de forma anual.

Respecto a las fuentes de información, el $75.7 \%$ refirió obtener información de medios digitales (Tabla $5)$.

\section{Discusión}

La popularidad de las dietas vegetarianas está aumentando, en especial en jóvenes y adolescentes, en el mundo occidental ${ }^{3,4,15}$. A diferencia de la frecuencia reportada en la literatura (3-5\%) en la población estudiada fue de $51,3 \%$. Esto puede explicarse por sesgo de selección. Al igual que en otros estudios, la mayoría eran mujeres $(84 \%)^{7}$. Un $4,6 \%$ eran veganos que, si bien es mayor a lo descrito en publicaciones recientes, en los últimos años se ha visto un aumento progresivo de su prevalencia ${ }^{7,17}$.
Las sociedades científicas han establecido que una dieta vegetariana, incluyendo las veganas, son adecuadas para todas las etapas de la vida si son bien planificadas y suplementadas ${ }^{1,5,17}$. Un 32,4\% de los encuestados, se hicieron vegetarianos entre los 12-18 años. Cabe destacar que de la muestra total solo un $42 \%$ había consultado a un profesional de la salud. A pesar de que existen diferentes estudios epidemiológicos que muestran beneficios de practicar una dieta vegetariana ${ }^{9,11-13}$, un patrón alimentario sin consejería adecuada puede resultar en déficit de vitaminas y micronutrientes con riesgos en la salud $\mathrm{d}^{3,10,14,17,18}$.

Es así como, el déficit de vitamina B 12 es frecuente en vegetarianos, ya que esta se encuentra casi exclusivamente en productos de origen animal y solo en algunas variedades de algas y hongos ${ }^{4,15,17,19}$. Inicialmente, aún con niveles plasmáticos adecuados de vitamina B12, se observa una elevación de la homocisteína, lo cual es un factor independiente e irreversible para enfermedad cardiovascular. La deficiencia de vitamina B 12 se manifiesta como anemia megaloblástica y alteraciones neurológicas ${ }^{4,11,15,20}$. Dado lo anterior, se recomienda que todos los vegetarianos, incluyendo los lacto-ovo-vegetarianos, deben recibir suplemento de vitamina $\mathrm{B} 12^{21}$. El 15,9\% de la población estudiada refirió presentar este déficit, pero el 52,9\% de la población total de vegetarianos, recibía suplementación con vitamina B 12.

Por otro lado, en las dietas vegetarianas predomina la ingesta de ácidos grasos poliinsaturados pertenecientes a la serie omega-6 (n-6) y es deficitaria la ingesta de omega-3 (n-3), lo que afecta la síntesis de ácido eicosapentanoico (EPA) y ácido docosahexaenoico (DHA). Numerosos estudios han demostrado que la ingesta $y$ concentración en plasma de EPA y DHA en lacto-ovovegetarianos y veganos, es menor que en omnívoros. Esto puede ser un inconveniente para la salud ya que ambos tienen un papel importante en las funciones neurológicas, cardiovasculares, cognitivas, entre otras. Es posible maximizar la conversión de ácido alfalinolénico (ALA) a EPA y DHA combinando un aumento en la ingesta de ALA y una disminución en la de ácido linoleico (LA), logrando un equilibrio óptimo entre n3/n-6. La ingesta de ALA se puede aumentar mediante el consumo de chia, linaza, nueces, aceite de canola y aceite de soya o en su defecto con algún suplemento ${ }^{17,20,22}$. En este estudio, un 5,7\% refiere suplementarse con omega 3, pero se desconoce su composición.

Respecto al calcio, los lácteos se consideran la principal fuente en la dieta. Los alimentos vegetales también son ricos en este mineral, pero presenta una menor biodisponibilidad por el alto consumo de fibra, ácido oxálico y ácido fítico ${ }^{8,19}$. El impacto de esto en la mineralización ósea está siendo estudiando ${ }^{20}$. Por otro lado, la baja biodisponibilidad de hierro, zinc y selenio 
en este tipo de dietas, hace que se deba disminuir la ingesta de fitatos, tanina y polioles que interfieren en su absorción, debiendo aumentar la ingesta de ácido cítrico $^{12,19}$.

En el ultimo tiempo ha existido alerta entre dieta vegetariana y posibles riesgo de accidente cerebrovascular, sin embargo no se han identificado aun los posibles nutrientes involucreados ${ }^{23}$.

En cuanto a las fuentes de información, al igual que en el resto del mundo, la mayoría de los encuestados $(75,7 \%)$ se informan a través de medios digitales y tan solo un $45,9 \%$ de profesionales de salud. Ahora bien, en una encuesta realizada el 2019 por Bettinelli et al a profesionales de la salud, demostró que estos no cuentan con los conocimientos adecuados acerca de las dietas vegetarianas, de sus consecuencias en la salud y respecto a consejería en las diferentes etapas de la vida $^{24}$, lo que hace fundamental la capacitación de los profesionales de salud en el tema.

Las motivaciones principales fueron medio ambientales $(91,9 \%)$ y animalistas $(72,9 \%)$, al igual que en la literatura ${ }^{5-9}$. Sin embargo, guías alimentarias e informes gubernamentales reconocen que las dietas basadas en plantas tienen efecto en el medio ambiente y en la salud ${ }^{6,17}$. Cabe destacar, que un $8,1 \%$ siguen este tipo de dieta como estrategia para controlar su peso y un 2,8\% de los vegetarianos presenta un Trastorno de Conducta Alimentaria; estos datos son interesantes y manifiestan la importancia de siempre investigar su presencia ante pacientes que deciden iniciar o mantienen este tipo de dietas.

En cuanto a los limitantes del estudio, la muestra incluye sólo a una universidad de la Región Metropolitana de Santiago, por lo que sus resultados no se pueden extender a la población universitaria chilena. Además hubo un porcentaje bajo de respuestas a la encuesta on line, pero similar a lo reportado en la literatura, pudiendo seleccionar a aquellos alumnos con mayor interés en el tema.

A pesar de estas limitaciones, este estudio exploratorio inicial contribuye al conocimiento de la utilización de dietas vegetarianas en universitarios. Son ne- cesarios futuros estudios multicéntricos de estudiantes de diferentes regiones para obtener resultados generalizables a toda la población de nuestro país.

\section{Conclusiones}

Existe un elevado porcentaje de vegetarianos y especialmente veganos entre los universitarios que contestaron la encuesta, lo que hace necesario que los profesionales de salud estén capacitados en esta área para asegurar una adecuada consejería y seguimiento. Estudios han demostrado una asociación protectora entre una dieta vegetariana y factores de riesgo de enfermedades crónicas no transmisibles; sin embargo la literatura internacional ha descrito un aumento en el riesgo de accidente cerebrovascular asociado a dietas vegetarianas ${ }^{25}$. Esperamos contribuir al conocimiento de la situación actual de jóvenes con respecto a las dietas vegetarianas, siendo un punto inicial y fundamento para futuras investigaciones en el tema.

\section{Responsabilidades Éticas}

Protección de personas y animales: Los autores declaran que los procedimientos seguidos se conformaron a las normas éticas del comité de experimentación humana responsable y de acuerdo con la Asociación Médica Mundial y la Declaración de Helsinki.

Confidencialidad de los datos: Los autores declaran que han seguido los protocolos de su centro de trabajo sobre la publicación de datos de pacientes.

Derecho a la privacidad y consentimiento informado: Los autores han obtenido el consentimiento informado de los pacientes y/o sujetos referidos en el artículo. Este documento obra en poder del autor de correspondencia.

\section{Conflicto de intereses}

Los autores declaran no tener conflicto de intereses.

\section{Referencias}

1. Brignardello GJ, Heredia PL, Ocharán SMP, Durán AS. Food knowledge of chilean vegetarians and vegans. Rev Chil Nutr. 2013;40(2):129-34.

2. Parker HW, Vadiveloo MK. Diet quality of vegetarian diets compared with nonvegetarian diets: A systematic review. Nutr Rev. 2019;77(3):144-60.

3. Schürmann S, Kersting M, Alexy U. Vegetarian diets in children: a systematic review. Eur J Nutr. 2017;56(5):1797-817.
4. Derbyshire EJ. Flexitarian Diets and Health: A Review of the Evidence-Based Literature. Front Nutr. 2017;3:1-8.

5. Martínez Biarge M. Niños vegetarianos , ¿ niños sanos? In: 3.0 LE, editor. AEPap Congreso de Actualización Pediatría 2019. Madrid; 2019. p. 65-77.

6. United Nations System Standing Committee on Nutrition. Dietas sostenibles para una población y un planeta sanos United Nations System Standing Committee on Nutrition ES Documento de debate. 2017; https://www. unscn.org/uploads/web/news/document/ Climate-Nutrition-Paper-SP-nov-2017WEB.pdf

7. Pfeiler TM, Egloff B. Examining the "Veggie" personality: Results from a representative German sample. Appetite. 2018;120:246-55.

8. Sebastiani G, Barbero AH, Borrás-Novel $C$, et al. The effects of vegetarian and vegan diet during pregnancy on the health of mothers and offspring. Nutrients. 2019;11(3):1-29.

9. Bravo JP, Ibarra CJ, Paredes MM. 
Compromiso neurológico y hematológico por déficit de vitamina B12 en lactante hijo de madre vegetariana. Caso Clínico. Rev Chil Pediatr. 2014;85(3):337-43.

10. Pilis W, Stec K, Zych M, Pilis A. Health benefits and risk associated with adopting a vegetarian diet. Rocz Państwowego Zakładu Hig. 2014;65(1):9-14.

11. Melina V, Craig W, Levin S. Position of the Academy of Nutrition and Dietetics: Vegetarian Diets. J Acad Nutr Diet. 2016;116(12):1970-80.

12. Dinu M, Abbate R, Gensini GF, Casini A, Sofi F. Vegetarian, vegan diets and multiple health outcomes: A systematic review with meta-analysis of observational studies. Crit Rev Food Sci Nutr. 2017;57(17):3640-9.

13. Gorczyca D. Nutritional Status of Vegetarian Children. Veg Plant-Based Diets Heal Dis Prev. 2017;529-47; http:// dx.doi.org/10.1016/B978-0-12-8039687.00030-7

14. Le Roy OC, Díaz San Martín X. Dieta vegetariana en la edad pediátrica. Gastroenterol Latinoam. 2010;21(1):9-14.

15. Elorinne AL, Alfthan G, Erlund I, et al. Food and nutrient intake and nutritional status of Finnish vegans and nonvegetarians. PLoS One. 2016;11(2):1-14.

16. Miniesterio de Medio Ambiente. Informe Final "Encuesta Nacional de Medio Ambiente 2018 ” Licitación N 608897 110-LE17 Dirección de Estudios Sociales (DESUC) del Instituto de Sociología, Universidad Católica. 2018;1-122; https:// mma.gob.cl/wp-content/uploads/2018/03/ Informe-Final-Encuesta-Nacional-deMedio-Ambiente-2018.pdf

17. Lemale J, Mas E, Jung C, Bellaiche M, Tounian P. Vegan diet in children and adolescents. Recommendations from the French-speaking Pediatric Hepatology, Gastroenterology and Nutrition Group (GFHGNP). Arch Pediatr. 2019;26(7):442-50.

18. Serralde-Zúñiga AE, Pasquetti-Ceccatelli A, Meléndez-Mier G. Micronutrimentos en vegetarianos. Rev Endocrinol y Nutr. 2005;13(1):33-8.

19. Mullee A, Vermeire LVB. Vegetarianism and meat consumption: A comparison of attitudes and beliefs between vegetarian, semi-vegetarian, and omnivorous subjects in Belgium. Appetite. 2017;114:299-305.
20. García-Maldonado E, Gallego-Narbón A, Vaquero MP. Are vegetarian diets nutritionally adequate? A revision of the scientific evidence. Nutr Hosp. 2019;36(4):950-61.

21. Pawlak R, Lester SE, Babatunde T. The prevalence of cobalamin deficiency among vegetarians assessed by serum vitamin B12: A review of literature. Eur J Clin Nutr. 2014;68(5):541-8.

22. Burdge GC, Tan S-Y, Henry CJ. Longchain n -3 PUFA in vegetarian women: a metabolic perspective.J Nutr Sci. 2017;6:1-8.

23. Lawrence MA, McNaughton SA. Vegetarian diets and health. BMJ. 2019;366:1-2.

24. Bettinelli ME, Bezze E, Morasca L, et al. Knowledge of health professionals regarding vegetarian diets from pregnancy to adolescence: An observational study. Nutrients. 2019;11(5):3-11.

25. Tong TYN, Appleby PN, Bradbury KE, et al. Risks of ischaemic heart disease and stroke in meat eaters, fish eaters, and vegetarians over 18 years of follow-up: Results from the prospective EPICOxford study. BMJ. 2019;366(1). 